\title{
“LUCHARÉ Y LUCHARÉ Y HASTA QUE LO CONSIGA NO PARARÉ. UNA APROXIMACIÓN AL TEMA DEL ABANDONO ESCOLAR A TRAVÉS DE UNA HISTORIA DE VIDA"
}

\author{
“I WILL FIGHT AND FIGHT AND I WILL NEVER STOP UNTIL I WILL GET \\ IT. AN APPROXIMATION TO THE SCHOOL DROPOUT TOPIC THROUGH A \\ HISTORY OF LIFE
}

Inmaculada Garrido Jiménez*

\begin{abstract}
El objetivo de este artículo es contribuir al estudio del fracaso escolar por medio del análisis de una historia de vida que por un lado cumple las condiciones y circunstancias que la literatura académica ha señalado como condicionantes del fracaso escolar, pero también usando la historia de vida en contrapunto y como desafío a esas condiciones tratando de analizar en su relato qué aspectos son los que le ha permitido a la protagonista superar todos esos condicionantes.
\end{abstract}

Palabras claves: Rendimiento académico, nivel de conocimientos, historia de vida, fracaso escolar, abandono escolar.

The aim of this article is to contribute to the study of school failure through the analysis of a history of life that, on one hand, it has got the conditions and circunstances that the academic literature has pointed as conditions for a school failure but at the same time using the history of life as a challenge of these conditions trying to analyze in the story which are the aspects that let the main character overcome all these conditions.

Key words: Academic performance, knowledge level, history of life, school failure, school dropout.

\section{Introducción}

En una sociedad como la actual, en continuo cambio y evolución, la gran diversidad tanto cultural como social existente hace que cada persona pese a disponer de las mismas oportunidades de acceso a la educación vaya forjando su camino y su personalidad hasta que llega a convertirse en una persona íntegra que se diferencia sustancialmente de otra.

Nuestras escuelas están repletas de alumnos a los que gratuitamente nos atrevemos a catalogar como "buenos" o "malos" atribuyendo este éxito o fracaso básicamente a su actitud hacia el estudio sin detenernos a pensar en cuáles podrían ser las verdaderas razones que llevan a este alumno a obtener esos resultados, a fracasar o triunfar académicamente.

En una investigación anterior sobre rendimiento escolar centré mi análisis en algunos de los factores que influyen en el alumnado: el contexto social, económico, cultural, familiar, el círculo de amistades, etc... ipero son verdaderamente estos los factores que influyen en la persona o hay otra serie de aspectos a considerar?
Para analizar cada uno de estos aspectos se trabajó con historias de vida debido al hecho de que dicha técnica aporta datos muy relevantes desde la perspectiva interna del propio informante, lo que otorga a la investigación un matiz de veracidad que no se obtiene con otras técnicas. El presente artículo tiene la intención de argumentar algunas de las conclusiones de ese trabajo.

\section{Diseño de la metodología}

Las historias de vida son una técnica de investigación cualitativa cuyo objetivo principal es el análisis de los relatos de una persona sobre su vida o momentos concretos de la misma (Martín, 1995), y también sobre los relatos y documentos extraídos de terceras personas, es decir, relatos y aportaciones realizadas por otras personas sobre el sujeto de la historia de vida (Perelló, 2009).

Como dice Pujadas (1992:47) el objetivo es mostrar el testimonio subjetivo de una persona en la que se recojan tanto los acontecimientos como las valoraciones que dicha persona hace de su

* Universidad a Distancia (UNED), Facultad de Educación, Departamento de MIDE I. España. Correo electrónico: inmagaji@ hotmail.com 
propia existencia. El investigador induce la narración, la transcribe y "retoca" el texto para ordenar la información del relato (Perelló, 2009). Además, compartiendo la afirmación de Díaz de Rada (2006), una historia de vida es una reconstrucción de la vida de una persona que parte de ella misma. La manera en que esta persona organice y priorice los hechos dice mucho sobre sus inquietudes y aspectos más significativos. De la misma forma siguiendo a Leite (2011) las historias de vida se basan en experiencias concretas de la persona entrevistada, mediante las que se pretende recuperar el sentido de la misma vinculándola a experiencias vividas de las personas (dentro de la subjetividad de la misma) y que nos permiten poner de manifiesto y revelar las técnicas de investigación cualitativa.

Las historias de vida nos permiten visualizar, entender e interpretar las voces que siempre han estado, pero los discursos dominantes de nuestra sociedad nos han imposibilitado ver (Cortés, 2011). De la misma manera, abarcan un amplio recorrido, ya que como afirma Leite (2011) su interpretación no tiene lugar en un momento concreto, sino que comienza prácticamente desde el momento en que nos empezamos a plantear interrogantes e inquietudes por investigar y analizar.

Este tipo de investigación cualitativa que se corresponde con lo que Hernández (2010) define como una forma de volverse hacia la subjetividad como conocimiento y el relato (o la biografía) podría considerarse como fuente para este conocimiento. Por ello, como indica Ocaña (2011), nos va a permitir conocer, investigar y analizar diferentes categorías de las experiencias de vida de las personas que han tenido un importante peso en la trayectoria.

\section{Acercamiento al concepto de rendimiento académico y fracaso o abandono escolar}

El concepto de rendimiento escolar ya ha sido estudiado. Así Jiménez (2000) lo define como nivel de conocimientos demostrado en un área o materia comparado con la norma de edad y nivel académico. Otros como Tonconi (2010) definen el rendimiento académico como el nivel demostrado de conocimientos en un área o materia, evidenciado por medio de indicadores cuantitativos, habitualmente expresados mediante calificación ponderada en el sistema vigesimal y, bajo el supuesto que es un "grupo social calificado" el que fija los rangos de aprobación para áreas de conocimiento determinadas, para contenidos específicos o para asignaturas. Según estas definiciones se entiende que el rendimiento académico es solamente un resultado dejando sin explicar cómo aprende el alumno.

Por otro lado, el rendimiento académico se entiende como logro y construcción de conocimientos en los estudiantes a partir de las intervenciones didácticas en una materia que son evaluadas mediante métodos cualitativos y cuantitativos (Zapata, De los Reyes, Lewis y Barceló, 2009).

Pero unidos a esta definición, otros aspectos deben ser tenidos en cuenta, como pueden ser la conducta del alumno, el esfuerzo, la atención en clase, asistencia, etc. De esta manera, el concepto de rendimiento se convierte en un criterio bastante subjetivo, amplio y susceptible de influencias externas al individuo y que, desafortunadamente, condicionará las posibilidades que pueda tener el niño tanto social como profesionalmente en el futuro.

A partir de los argumentos anteriores, podemos deducir que rendimiento académico no es solamente una capacidad innata que tiene el alumno, sino que es un cúmulo de muchos factores tanto intrínsecos (motivación, ganas de aprender y superarse, esfuerzo) como extrínsecos como contexto sociocultural y familiar en el que se desarrolla el estudiante y que son los que forjan una aptitud y actitud hacia la adquisición de conocimientos y desarrollo de competencias para conseguir un logro académico con éxito.

Teniendo en cuenta estos aspectos el artículo que nos ocupa pretende analizar la historia de Cristina (1) de manera que sea posible prestar atención a las circunstancias asociadas.

\section{Dimensiones en la historia de vida de Cristina que influyen en su rendimiento escolar}

En el análisis de la historia de vida de Cristina se han identificado distintas dimensiones que, desde el punto de la literatura académica, son determinantes del fracaso escolar. La historia de Cristina, sin embargo, ofrece un contrapunto a este determinismo, ya que aún compartiendo todos estos factores, su historia no termina en un fracaso, sino en todo lo contrario como se puede observar a continuación.

\section{Candidata al fracaso escolar}

Cristina tiene una historia de vida bastante complicada. Su infancia ha estado llena de idas y 
venidas a diferentes casas de acogida en las que no encontraba ni el cariño ni la motivación adecuada hacia el estudio. De hecho ella misma afirma que en los centros de acogida existían las figuras de tutores o profesores que se encargaban de ayudar a estudiar, pero que en realidad no lo hacían:

Allí en el centro, si querías estudiar podías, pero no lo hacía nadie; imagínate cada uno de su padre y de su madre... y aunque se supone que ellas te iban a ayudar no era así solamente ayudaban a los listos; a mí nadie me ayudaba yo hacía lo que podía (Cristina).

Es evidente que el aspecto contextual y social juega un papel muy decisivo a la hora de determinar tanto las oportunidades como los medios y recursos necesarios para poder disfrutar de los estudios.

Sí, sí, y cuando mi padre se separaba pues íbamos a vivir a casa de mis abuelas porque él decía que no podía estar atendiendo a sus hijos, bueno... es que mi padre es alcohólico y no podía estar atendiendo a seis niños entonces nos dejaba en casa de unas abuelas o de otras y ya cuando volvía con sus mujeres era cuando ya si nos cogía de nuevo (Cristina).

Según Valdés y Urías (2010), cuando se ha estudiado la influencia de la familia en el logro escolar, se ha comprobado el efecto de dos grupos de factores. Por un lado, los llamados como de insumo o estructurales (condición socioeconómica, nivel de escolaridad de los padres y recursos para el estudio, entre otros); y por otro lado, los conocidos como procesuales (expectativas de los padres, dinámica familiar y participación en la educación de los hijos, entre otros). Es necesario hacer una mención explícita a los factores que afectan al funcionamiento familiar (Avanzini, 1995; Valdés, Esquivel y Artiles, 2007). Siguiendo a Valdés y Urías (2010), el mal funcionamiento de la familia trae consigo una mala participación de los padres en la educación de sus hijos, su crianza, la supervisión del aprendizaje y la comunicación con la escuela.

A mi padre no le importaba cómo estábamos... él pasaba de nosotros ni se preocupaba por lo que ocurría en el colegio cuando estaba con nosotros así que cuando estábamos en las casas..., pues imagínate (Cristina).

Cristina reconoce que no ha tenido un clima familiar adecuado, que trasladarse de casa de acogida en casa de acogida no es un buen ambiente para una niña. Además, percibe que el hecho de que tanto ella como sus hermanas estuvieran en la casa de acogida no estaba bien considerado en el centro escolar.

Nosotras éramos las de la "resi" nadie quería estar con nosotras y yo creo que además..., pensaban que éramos tontas o no podíamos sacar buenas notas. O por lo menos yo me sentía así (Cristina).

A pesar de que la literatura académica identifica un ambiente familiar de clase media con el interés por los estudios de los hijos, el caso de Cristina ofrece una excepción. Su padre disfrutaba de una buena situación laboral, aunque ella cuenta que:

mi padre en el momento en que nos trasladaban pues primero eran sus cosas y luego ya cuando se acordaba nos matriculaba (Cristina).

Cristina perdió a su madre con tan solo dos años y su padre tuvo varias parejas después, pero: cuando se separaba pues íbamos a vivir a casa de mis abuelas porque él decía que no podía estar atendiendo a sus hijos (Cristina).

Finalmente ingresó a sus hijos en un centro de acogida. Cristina pasó de ser una niña de padres de clase media a ser una de muchas en un centro de acogida:

En el centro este no tengo muchos recuerdos y los pocos que tengo son horribles... Nos pegaban y luego cuando te portabas mal nos... había un patio trasero con una verja y eso era...vamos que en cualquier momento te podías despeñar por ahí porque estaba el río abajo y cuando te portabas mal por la noche te sacaban allí con todo apagado, a lo mejor estábamos 4 o 5 niños ahí llorando porque se supone que te portabas mal y ese era el castigo que te hacían o te pegaban o, simplemente, si ellas estaban hablando y 
claro no querían... porque claro, estaban las de día y las de noche y si tú tenías pesadillas ya te podías desgañitar llorando que no te hacían caso hasta que ya de puro agotamiento te quedabas dormida, muy mal, muy mal... (Cristina).

Afirma que los responsables del Centro se preocupaban de satisfacer las necesidades básicas de los niños, pero nada más.

Ellas te cuidaban, te alimentaban, pero nada más éramos muchos y bueno, luego jamás te besaban, ni abrazaban no te daban cariño no sé si era algo tabú o es que simplemente porque están muy entregadas a su vida y saben que van a pasar muchos niños por su vida y prefieren mantener cierta distancia entonces yo siempre he tenido muchos problemas para relacionarme (Cristina).

No se puede pasar por alto las historias de los hermanos y hermanas. Los dos hermanos mayores fueron enviados a un centro de menores $\mathrm{y}$, en palabras de Cristina, fueron los que peor lo pasaron porque tuvieron que convivir con chicos muy problemáticos, viviendo con el sentimiento de estar solos sin el apoyo del padre. Las dos hermanas se quedan embarazadas con tan solo 13 y 14 años y son rechazadas tanto por su familia como por los responsables del centro de acogida. Solicitaron la emancipación judicial, les fue concedida y a partir de ahí inician sus vidas solas.

Finalmente, se refiere a la historia del hermano menor de la siguiente manera:

Él estuvo también en el Centro de Acogida conmigo y pasó la misma situación que yo, lo que pasa es que él siempre ha sido como más débil, entonces, incluso mi tía, la que te digo que siempre se portó bien con nosotros, le acogió y estuvo viviendo con ella porque la verdad es que era una mujer buenísima y le trató como un hijo, pero ya se le veía que estaba con malas compañías, ya se portaba fatal, tenía problemas con todos los vecinos, con todos los chicos y nunca ha querido ataduras ni que le impusiesen normas, ha vivido siempre al margen de todo, entonces, ya daba muchos problemas, robaba ya maltrataba a todo el mundo a familia y todo, nos ha robado, nos ha pegado y ya llega un momento en el que decides... (Cristina).

Según Salomón (2011), la falta de atención por parte de los padres, especialmente durante la educación primaria, provoca que los niños no se sientan motivados en la escuela, y por consiguiente, consigan un rendimiento menor. Cristina dice de su padre:

\begin{abstract}
No se enteraba de nada o no se quería enterar entonces... es que nunca ha tenido interés porque sus hijos llegasen a algo como la mayoría de los padres que quieren que sus hijos estudien y todo yo me acuerdo... (Cristina).
\end{abstract}

La función socializadora que realizan los padres es muy importante. La relación entre padres e hijos, a su vez, depende del estilo de interacción parental estructurado por los padres y del clima social que se haya establecido en la organización y dinámica del núcleo familiar:

Los estilos de interacción familiar y el clima social familiar son variables que explican y predicen el desarrollo de habilidades sociales; por eso, en niños en quienes se observaba un repertorio básico para iniciar y mantener situaciones sociales, se establece una relación entre el estilo parental equilibrado y con los componentes de desarrollo (procesos de desarrollo personal, que son fomentados en el núcleo familiar), relaciones (canales de comunicación y expresión propiciados en la familia) y estabilidad (estructura y organización de la familia y grado de control que los padres ejercen sobre sus hijos e hijas) del clima social familiar (Isaza y Henao, 2010).

Se observa como este aspecto tiene una gran incidencia en Cristina que se define de la siguiente manera:

"yo era súper retraída... pero es que nunca me habían tenido en cuenta así que cuando estaba con los otros niños pues me quedaba callada y así pasaba desapercibida... como en mi casa. Bueno y de mi hermano no te digo nada... él llamaba la atención haciendo cosas malas" (Cristina).

Hace tiempo, autores como Himmel (1984) analizaron las expectativas y aspiraciones de los 
padres respecto del nivel educacional de sus hijos. Las expectativas se refieren al nivel educacional que los padres creen que sus hijos debieran tener. Las aspiraciones se refieren al nivel educacional que los padres desean que sus hijos alcancen. Este interés de la familia se demuestra valorando las tareas que se desarrollan en el colegio. Es evidente que en familias donde se valoren positivamente los trabajos desempeñados en la escuela se motivará en mejor medida al alumno que si no se le da ningún tipo de valoración o incluso si se valora negativamente el esfuerzo y los logros conseguidos.

El interés de los padres por el proceso de enseñanza aprendizaje de los hijos es fundamental para que se sientan motivados y reconocidos. Brown afirma que cuando los padres participan en la educación se obtienen beneficios, tanto para los padres como para el niño, ya que frecuentemente mejora la autoestima del niño, ayuda a los padres a desarrollar actitudes positivas hacia la escuela y les proporciona a los padres una mejor comprensión del proceso de enseñanza (1989:1).

Es evidente que una de las razones más poderosas que tiene el alumno para trabajar en la escuela es el sentido de estar enorgulleciendo a sus padres o que corresponda a las esperanzas depositadas en él. Si comprende que trabajando bien no causa ninguna alegría o sacando malas notas no provoca ningún disgusto familiar, llegará a desentenderse por los estudios.

Por ello, las creencias y conductas de los padres, $y$ las percepciones que el hijo tenga acerca de estas y de las expectativas de sus padres, es una dimensión relacionada con el éxito escolar. Es decir, si el padre cree y espera que su hijo tenga éxito en la escuela, esto va a influir en la formación de las aspiraciones académicas del hijo, y se esperaría que tuviese un alto aprovechamiento académico. Por otra parte, si el padre no tiene expectativas educativas, o no demuestra conductas que motiven al niño a desempeñarse con éxito en la escuela, es posible que el hijo tenga un bajo rendimiento escolar.

Hay que decir que el caso de Cristina es especial, su padre no siente el deseo de que sus hijos estudien. No todos los hogares cuentan con las mejores situaciones tanto económicas como personales para inculcarles unos buenos valores educativos a los hijos, pero, a pesar de que el padre posee un nivel cultural medio, se desentiende tanto de ella como de sus hermanos y se vieron obligados a tener que sobrevivir en un Centro de Acogida donde el nivel y las expectativas de futuro para esos niños no eran muy altas. Como ella bien afirma, su padre retiró la pensión de estudios tanto a ella como a su hermano viéndose obligados a trabajar para costeárselo, así que esto nos da una idea del interés que tenía el padre por sus hijos.

Cristina misma manifiesta que en ningún momento de su vida encontró apoyo familiar ni ánimo por parte de nadie:
¡Uy! fatal yo es que no tenía base, con lo cual a mí me costaba horrores, me pasé la EGB y el Bachiller sin atender en clase, yo estaba totalmente ida, de hecho, me ponía siempre al final, tenía pánico a hablar en público, pánico a salir a la calle, porque pensaba que la gente me miraba y se reía de mí, tenía la autoestima por los suelos porque yo vivía con mi abuela que estaba muy amargada y nunca se portó bien en el sentido de que siempre estaba menospre- ciándome y todo porque estaba amargada (Cristina).

El bajo rendimiento académico empieza a tener un significado desde el momento en que se inicia la educación, cuando el que aprende no da la respuesta adecuada a las normas ni a los tiempos del que enseña, pero es curioso señalar que a lo largo del tiempo, en vez de resolverse el problema, este se ha ido volviendo más serio.

Las expectativas de futuro que los padres tengan de sus hijos son factores muy influyentes en ellos, ya que en padres en los que se espera que los hijos estudien y se les motiva para ello el niño está más predispuesto a ello, e incluso, se siente en obligación de hacerlo por su padre, pero en casos en los que las expectativas de futuro no son tan ambiciosas, o incluso, los padres no son buenos ejemplos a seguir el niño se conformará con metas más modestas.

Por otra parte, el relato de Cristina nos deja ver el papel de la escuela de una manera ambivalente. Por un lado, critica el proceso de selección de acceso a la escuela, siendo un método muy discriminatorio del que se valían por la falta de supervisión por parte de las autoridades y por el hecho de tratarse de niños sin padres.

Primero te hacían un test de inteligencia para ver dónde te llevaban porque había 
niños de todo tipo, allí no era como ahora allí entraban todos los niños que tenían problemas con los padres, por maltrato o por fallecimiento de los padres o niños que no los querían los padres porque tenían algún retraso y ponían cualquier excusa o simplemente los abandonaban y los metían allí, entonces, te hacían un test para ver el nivel, realmente ellos te decían que era para ver si eras tonto (Cristina).

Para los niños que no tenían la suerte de superar el test, les esperaba una educación en el mismo centro de acogida.

Claro no te llevaban al cole tenías allí a un educador y los educaban allí, pero sin sacarlos [...] sí, sí, tenían algo parecido a lo que tenemos ahora que son los equipos de orientación, pues algo parecido [...] Bueno si allí estudiabas, bueno no había ningún niño que no hiciese nada todos estaban estudiando lo que pasa que a los que tenían un coeficiente intelectual por debajo de la media pues no se molestaban en matricularles (Cristina).

En el siguiente epígrafe vamos a ver el papel importante y positivo que jugaron algunos profesores a la hora de apoyar a Cristina a desafiar una trayectoria que la conducía al fracaso escolar.

\section{Desafío y éxito}

En Cristina se pueden ver cómo se dan los condicionantes que la literatura académica ha definido como detonantes para un fracaso escolar seguro, pero en este caso ella va sorteando esas barreras o condicionantes para abrirse paso y llegar a conseguir su meta.

Un aspecto que tiene gran influencia en el éxito o fracaso escolar de una persona tiene que ver con sus propias expectativas de futuro y su autoestima. El apoyo que recibe de la familia interfiere en la persona haciendo que uno mismo se desarrolle de una manera u otra.

En el caso de Cristina el hecho de que su abuela la menospreciase constantemente, aunque la hacía mucho daño, provocaba a la vez que ella luchara con más fuerzas por conseguir lo que se proponía. Seguramente, este deseo por romper con las expectativas negativas, en este caso de la familia, fueron las que le llevaron a volcarse más en sus propósitos y esforzarse más y más por llevar la contraria a sus familiares.

Ella misma tiene claro que para poder ser algo en la vida hay que estudiar y ese pensamiento es el que la mantiene alerta y con ganas de tirar para adelante a la vez que se ve empujada por demostrarles a los demás que ella es capaz de lo que se proponga.

Había que estudiar y hacer una carrera y entonces, pues yo sabía que sin estudios no vas a ningún sitio que era la única manera de encontrar un buen trabajo porque yo no estaba dispuesta a meterme de hostelera porque hombre, eso está bien para gente que no tiene otras opciones pero... (Cristina).

El clima y contexto social en el que crece Cristina hace que tenga una percepción de sí misma distorsionada a la vez que negativa que poco a poco va a cambiar radicalmente por el deseo de conseguir su propósito. El menosprecio de su familia fue un aspecto que le hizo reaccionar en contra de los que pensaban mal de ella y demostrarles que podía conseguirlo:

Es que yo para ellos siempre he sido muy jorobada y a mí bastaba que mi abuela me dijese esto: "es muy difícil, no lo vas a conseguir" para que yo me empecinase más en ello y bueno al final también un poco por contradecir a los que me rodeaban pues hice lo que hice (Cristina).

Ligado a lo anterior cobra especial importancia la motivación. Núñez (1996) afirma que la motivación no es un proceso unitario, sino que abarca componentes muy diversos que ninguna de las teorías elaboradas hasta el momento ha conseguido integrar, de ahí que uno de los mayores retos de los investigadores sea el tratar de precisar y clarificar qué elementos o constructos se engloban dentro de este amplio y complejo proceso que etiquetamos como motivación.

Tradicionalmente, se han distinguido los aspectos cognitivos y los afectivo-motivacionales a la hora de estudiar su influencia en el aprendizaje escolar y en el rendimiento académico. Autores como Cabanach et al. (1996:9) opinan que Se puede afirmar que el aprendizaje se caracteriza como un 
proceso cognitivo y motivacional a la vez, por eso, en la mejora del rendimiento académico se deben considerar tanto los aspectos cognitivos como los motivacionales. Para que una persona aprenda es necesario "poder" hacerlo, es decir, tener las capacidades, los conocimientos, las estrategias y las destrezas necesarias (componentes cognitivos), pero además hay que "querer" hacerlo, tener la disposición, la intención y la motivación suficientes (componentes motivacionales) (Núñez y GonzálezPumariega, 1996). Eruditos en este tema como Paris Lipson y Wixson (1983), Pintrich (1989), Pintrich y De Groot (1990) defienden que para obtener buenos resultados académicos, los alumnos necesitan poseer tanto "voluntad "(will) como "habilidad" (skill) (G. Cabanach et al., 1996), lo que conduce a la necesidad de integrar ambos aspectos.

Cristina tenía la voluntad y el querer hacerlo, pero le faltaban otros aspectos esenciales como son los emocionales que en muchas ocasiones son más importantes que los primeros si lo que se pretende es obtener buenos resultados.

Por otro lado, muy relacionado con la motivación se sitúa la autoestima y concepción que tenga uno de sí mismo, ya que hace que uno se sienta capaz o no de conseguir lo que se proponga. Otra vez más Cristina demuestra que aunque su autoestima se encontraba por los suelos el deseo de conseguir su propósito le hizo superarlo y conseguirlo: me propuse ser maestra y mira lo conseguí. Para mí eso fue lo mejor porque lo conseguí yo sola... y me costó, pero ya lo soy y muy orgullosa estoy (Cristina).

Seguramente el tesón y motivación que tenía la protagonista por llegar a ser alguien en la vida fue lo que la empujó a conseguir su objetivo.

Otro de los aspectos que hacen que Cristina supere el gran desafío que le plantea la vida es el gran sentido de la responsabilidad que la viene caracterizando desde pequeñita.

Reconoce que aunque la situación en su casa le permitía no ir al colegio o prescindir del estudio, su sentido de la responsabilidad siempre le ha obligado a estudiar dentro de la medida de lo posible.

yo siempre he tenido un sentido de la responsabilidad increíble porque ya te digo que yendo a $3^{\circ}$ y sin material porque mi padre no nos compraba material porque yo pasaba pues con lo que me dejaban los maestros y yo me iba sola. [...] Yo me levantaba a las 8 de la mañana sola, mi hermana se quedaba viendo los dibujos animados y yo me iba sola al colegio. [...] Fíjate que yo me ponía el despertador y todo para ir al cole, y mientras mis hermanas se iban por ahí yo me iba al cole porque sentía que tenía que estudiar y estar allí... (Cristina).

Como niña que era se puede deducir que este sentido tan grande de la responsabilidad le haría caer en algunas ocasiones en el deseo de abandonar y dirigirse hacia los modelos que tenía presentes que eran el de sus hermanos, pero, por el contrario, supo mantenerse firme en sus intenciones y supo encontrar en cada momento de flaqueza las fuerzas suficientes para volver a luchar por sus deseos. Por esto ella afirma: tenías que luchar por lo que querías por ti sola (Cristina).

Además, es evidente que el clima y el contexto en el que uno se desarrolle juega un papel importantísimo. El hecho de que una persona se desarrolle en un clima y contexto favorable al estudio condiciona su percepción de la educación y, por tanto, supone que tenga más posibilidades de conseguir el éxito escolar.

Es probable que las trayectorias de sus hermanos le dieran un ejemplo que lo que no se debía hacer $\mathrm{y}$, por eso, siempre ha intentado alejarse de esos modelos y construir su propio estilo; el que ella pensaba que era mejor. Los hermanos, aunque no eran buenos modelos para ella tuvieron una repercusión positiva. El hecho de ver cómo se desencadenaban las trayectorias y decisiones de los hermanos hizo que madurase de manera acelerada identificando estos patrones como negativos y haciendo que se alejase de ellos: yo es que veía a mis hermanas... y claro... yo eso no lo quería... (Cristina).

Por otro lado, el profesor juega un papel importantísimo en el proceso de enseñanza aprendizaje de los alumnos. El rol de los profesores en las aulas ha ido cambiando a lo largo del tiempo, adquiriendo cada vez más un papel de facilitadores de los aprendizajes y no de meros transmisores de contenidos (Del Moral y Villalustre, 2010; Mato, 2010).

Se entiende que los maestros tienen obligaciones hacia sus alumnos basadas fundamentalmente en ayudarles a aprender cosas valiosas en el contexto del aula y la escuela y aunque el trabajo del docente no es el de ser un psicólogo, este en ocasiones, debido a su preocupación por los niños, la simpatía, al afecto y al vínculo que se crea entre alumno-profesor se puede ver obligado a "ejercer" ese papel. El profesor 
no se limita a transmitir conocimientos, sino que en algunas ocasiones el afecto, la simpatía o la necesidad de ayuda que una persona puede demandar puede ser dado por él. Cristina hace alusión a maestros que le dejaron huella por el apoyo que le ofrecieron a la hora de conseguir material escolar, pero no por haberla apoyado moral o psicológicamente aspecto que tuvo que superar ella misma.

\section{yendo a $3^{\circ}$ y sin material porque mi padre no nos compraba material porque yo pasaba... pues... con lo que me dejaban los maestros y yo me iba sola (Cristina).}

\section{Conclusiones}

La historia de Cristina es claramente una ruptura con los condicionantes que la literatura académica considera como factores que conducen a un fracaso escolar.

Poco a poco, ella misma ha sabido sortear los diferentes obstáculos con los que se ha ido encontrando a lo largo de su vida para conseguir su máximo deseo: ser algo en la vida.

Pero en toda esta historia se puede deducir el papel que juega la escuela y, por eso, desde un enfoque intercultural basado en aspectos como la igualdad de oportunidades o equidad se extraen conclusiones como que en el caso de Cristina no fue el enfoque intercultural el que imperó y condujo su trayectoria escolar. La suerte que tuvo al aprobar un test de inteligencia le abrió las puertas de acceso a la escuela, ya que de lo contrario se hubiera tenido que quedar en el centro de acogida viendo como las posibilidades de llegar a ser algo se veían mermadas.

Por otro lado, en este tipo de enfoque es importante la práctica educativa y el papel del profesor. En este caso, Cristina hace alusión a que ellas "eran las de la resi" y ese aspecto no se tomaba en cuenta por los profesores para intentar cambiarlo. Ese desinterés por parte del profesorado hace que uno de los agentes esenciales de la escuela como es el profesor rompa con las premisas de un enfoque intercultural, ignorando la igualdad de oportunidades y la equidad como principio fundamental en su práctica. Como afirma Aguado (1991) una educación que pretenda favorecer la interculturalidad debe promover prácticas educativas dirigidas a todos y cada uno de los miembros de la sociedad en su conjunto, y no solamente a los alumnos de origen inmigrante .

Podemos decir que el caso de Cristina es un guiño a los diferentes condicionantes que según muchos autores desde años han considerado como causantes de un fracaso escolar. Pero aquí se ha visto cómo se han salvado muchos obstáculos con los que se ha encontrado la protagonista rebelándose en todo momento contra los prejuicios y estereotipos con los que se le asociaba con el único objetivo de conseguir lo que más anhelaba desde pequeña: ser maestra y ser algo en la vida pese a los prejuicios que existieran sobre ella.

\section{Referencias Citadas}

Avanzini, Guy

1995 El fracaso escolar. Barcelona: Herder.

Brown, P. C.

1989 "Involving Parents in the Education of their Children" [Digests]. Disponible en: http://www.ed.gov/ databases/ ERIC_Digests/ed308988.html. como generadora de información en la reconstrucción de historias de vida. II Jornadas.

Cortés, P.

2011 El Sentido de las historias de vida en investigaciones socioeducativas.Una revisión crítica en Hernández, Sancho y Rivas (coord.). Historias de Vida en Educación. Biografías en Contexto. ESBRINA-RECERCA, Universidad de Barcelona, $N^{\circ}$ 4. Pp. 68-74.

Del Moral, M. E.; Villalustre, L.

2010 Formación del Profesor 2.0. Desarrollo de competencias tecnológicas para la escuela 2.0. Magister, 23, 59-69.

Díaz de Rada, Ángel

2006 Etnografia y Técnicas de Investigación Antropológica. Madrid. Universidad Nacional de educación a distancia.
Fernández Enguita, M.; Mena, L.; Riviére, J. 2010 Fracaso y abandono escolar en España, Fundación "la Caixa", Barcelona.

González Cabanach, R.; et al.

1996 Psicología de la instrucción. Vol. I: Aspectos históricos explicativos y metodológicos. Barcelona, E.U.B.

Hernández, F.

2010 Las historias de vida en el marco del giro narrativo en la investigación en Ciencias Sociales: los desafíos de poner biografías en contexto. En F. Hernández, J. M. Sancho Y J. I. Rivas (ed.), Historias de vida en educación: biografías en contexto (13-22). Barcelona: Universidad de Barcelona.

Himmel, E.; Maltes, S.; Najluf, N.; Nicolás P. Gazmuri, Arancibia 1984 Análisis de la influencia de factores alterables del proceso educativo sobre la efectividad escolar. Universidad Católica de Chile. Santiago.

Isaza, L.; Henao, G. C.

2010 El desempeño en habilidades sociales en niños, de dos y tres años de edad, y su relación con los estilos de 
interacción parental. Electronic Journal of Research in Educational Psychology, 8(3).

Jiménez, M.

2000 Competencia social: intervención preventiva en la escuela.Infancia y Sociedad. 24, pp. 21-48.

Leite Mendez, A. E.

2011 Historias de Vida de Maestros y Maestras. La

Martín García, A. V.

1995 Fundamentación Teórica y Uso de las Historias y Relatos de Vida como técnicas de Investigación en Pedagogía Social. Aula, 7, 41-60.

Mato, A.

2010 Las escuelas y los maestros de primeras letras (s. XIX) Magister, 23, 19-23.

Núñez, J. C.; González-Pumariega, S.

1996 Motivacion y aprendizaje escolar. Congreso Nacional sobre Motivación e Instrucción. Actas, pp. 53-72.

Ocaña, A.

2011 La entrevista biográfica biográfica: análisis de esta herramienta personal. Tesis Doctoral, Universidad de Málaga.

Paris, S. G.; Lipson, M. Y.; Wixson, K.

1983 Becoming a strategic reader. Contemporary Educational Psychology, 8, 293-316.

Perelló, S.

2009 Metodología de la Investigación Social. Madrid: Dykinson.

Pintrich, P. R.

1989 The dynamic interplay of student motivation and cognition in the college classroom. En C. Ames y M. L. Maher (eds.): Advances in motivation and achievement (vol. 6). Greenwich, CT: JAI Press.

Pintrich, P. R.; De Groot, E. V.

1990 Motivational and self-regulated learning components of classroom performance. Journal of Educational Psychology, $82,33-40$.

Pujadas Muñoz, J. J.

1992 El Método Biográfico: El Uso de las Historias de Vida en Ciencias Sociales. Madrid. Colección Cuadernos Metodológicos. Centro de investigaciones sociológicas.

Tonconi Quispe, J.

2010 Factores que Influyen en el Rendimiento Académico y la Deserción de los Estudiantes de la Facultad de Ingeniería Económica de la UNA-Puno (Perú). Cuadernos de Educación y Desarrollo, vol. 2, $\mathrm{N}^{\mathrm{o}}$ 1, enero. Universidad de Guadalajara, Los Lagos, Jalisco, México, p. 45.

Valdés, A.; M. Urías

2010 "Familia y logro escolar", en A. Valdés y J. Ochoa (eds.), Familia y crisis. Estrategias de afrontamiento, México, Pearson.

Valdés, A.; Esquivel, L.; Artiles, K.

2007 Familia y desarrollo. Estrategias de intervención en terapia familiar. México: Manual Moderno.

Zapata, L.; De Los Reyes, C.; Lewis, S.; Barceló, E.

2009 Memoria de trabajo y rendimiento académico en estudiantes de primer semestre de una universidad de la ciudad de Barranquilla. Psicología Desde El Caribe, 23, 66-82. 
